\title{
Diabetes Health Education: Nurses' Knowledge of Essential Components at a Rwandan Hospital
}

\author{
Vedaste Bagweneza ${ }^{1 *}$, Priscille Musabirema ${ }^{1}$, Marie Josée Mwiseneza ${ }^{1}$, Anita Collins ${ }^{2}$, Busisiwe Rosemary Bhengu ${ }^{2}$ \\ ${ }^{1}$ School of Nursing and Midwifery, University of Rwanda, College of Medicine and Health Sciences, Kigali, Rwanda \\ ${ }^{2}$ Rory Meyer's College of Nursing, New York University, New York, USA
}

*Corresponding author: Vedaste Bagweneza. School of Nursing and Midwifery, College of Medicine and Health Sciences, University of Rwanda, Remera Campus, 11 KG 47, Kigali, Rwanda. E-mail: vedavich1@yahoo.fr

\begin{abstract}
\section{Background}

Diabetes mellitus type 2 (T2DM) is the most prevalent form of diabetes that has continued to increase worldwide over the past decades. The cornerstone of T2DM management is education on self-management. Evidence shows that nurses have insufficient knowledge of the content of T2DM patient education.

Objective

To assess nurses' knowledge of health education content for T2DM patients, and to establish the relationship between their education and knowledge level of T2DM health education.

Methods

A descriptive cross-sectional design and total population sampling strategy were used to recruit nursing staff at a medical/surgical unit. Fifty-one nurses at the referral hospital of Rwanda completed the self-administered questionnaire. Descriptive statistics were used for data analysis.

Results

Nurses exhibited poor knowledge of diabetes health education. There was no significant relationship between the nurses' level of education and diabetes health education knowledge $(\mathrm{p}=0.102)$.

\section{Conclusion}

Nurses had good general knowledge of diabetes, though a low level of knowledge of diabetes health education in this low-resource setting. Hospitals equipped with a T2DM protocol and appropriate staff training would likely improve the nurses' knowledge and patient care outcomes.
\end{abstract}

Rwanda J Med Health Sci 2019;2(2):172-177.

\section{Keywords: Type 2 diabetes mellitus, nurses, knowledge, patients' self-management education}

\section{Background}

The global prevalence of Type Two Diabetes Mellitus (T2DM), a non-communicable disease (NCD), has risen in recent decades[1] and beckons healthcare providers (HCP) to take an active role in preventive measures and management. In 2017, 425 million people worldwide had diabetes [1] with an estimated 1.6 million deaths, and disproportionally affecting low-to-middle-income countries (LMIC).[2] In subSaharan Africa (SSA) in 2015, there were 14.2 million people with diabetes[1] and Rwanda had a prevalence of $3.2 \%$, with nearly 2000 deaths.[3] Diabetes is predicted to increase to 629 million by 2045 globally[1] and is one of the four priority NCDs targeted by the Sustainable Development Goals (SDGs).[2] In addition to infectious diseases, African healthcare needs are now focused on NCDs, [4] as countries transition at the demographic, sociocultural, and economic level. [5] Detection, screening and treatment are the leading solutions to curatively manage diabetes, [4] though many countries face significant challenges to meet local healthcare needs.[6] A systematic review in SSA (1999-2011) reported that the existing and potential solutions are complex,[7] requiring a comprehensive broad-based healthcare system.[5] Diabetes is a multiorgan disease and the majority of cases in SSA are undiagnosed,[1] due to lack of screening and access to healthcare facilities, and limited training of healthcare providers (HCP).[6] Other challenges include lack of care protocols, research of African populations, and inconsistencies between urban versus rural, and public versus private patients. [6]

Due to the numerous challenges, HCP have had to find solutions that work within their local context. Africans with diabetes are at higher risk for serious microvascular complications, whereas highincome populations commonly have macrovascular complications. [6] Common microvascular symptoms seen in SSA include cataracts, retinopathy, neuropathy, microalbuminuria and nephropathy,[6] particularly 
in patients with poor glycemic control and late-stage disease.[7] Macrovascular symptoms include stroke, heart disease, and peripheral vascular disease.[6] In addition, there is a significant interaction between diabetes and infectious diseases such as HIV.[7]

The relatively low level of awareness and understanding of diabetes and its complications among HCP and patients, combined with the previously mentioned challenges, results in patients not receiving appropriate care in the early stages when they need it the most.[5] Since nurses are the main HCP, questions arise about nursing knowledge of diabetes and health education. The five main categories for T2DM health education are known as the Five M's: Monitoring blood glucose, nutritious Meals, Move to increase physical activity, Medications, and More, such as foot care.[8] As the primary workforce in healthcare, nurses are in a prime position to provide diabetes testing and health education.

Nurses need access to evidenced-based T2DM health information, including care protocols, in order to improve health outcomes. According to numerous studies, nurses' knowledge of T2DM is limited. A study conducted in Ghana found that nurses had poor knowledge of the nutritional management of diabetes,[9] and other studies indicated diabetic diets are often improperly balanced.[10,11,12] Two other studies, in Rwanda,[13] and Zimbabwe,[14] reported nurses lacked knowledge of pain management. Other studies reported that nurses lack updated resources and insufficient knowledge, and many patients do not receive any formal diabetes education $[14,15,16]$ Conversely, a study in Nigeria reported nurses had good knowledge of HIV,[17] a topic with specific WHO protocols, regular nationwide training and international support.

Inappropriate diabetic patient education is common and constitutes the contributing factor in patients' poor knowledge and overall poor outcomes. Lack of evidence-based knowledge and the ability to provide appropriate care increases the risk of morbidity and mortality, and out-of-pocket expenditure, sometimes resulting in catastrophic and impoverishing health costs affecting individuals and their families. [18] These costs are extremely challenging for people without health insurance. Nurses educate patients with T2DM when they encounter them; however, there is limited information on T2DM care and availability of a recent protocol in Rwanda. Therefore, this study aimed to assess the nurses' knowledge of diabetic care, and the relationship between their education and knowledge level of T2DM health education.

\section{Methods}

\section{Design}

A cross-sectional study design was used to assess the knowledge of staff nurses likely to be providing T2DM care on two hospital units. The study was conducted at the 480-bed University Teaching Hospital of Butare
(CHUB), in the Southern province, Huye District, Rwanda during March and April 2017.

\section{Participants' recruitment}

All fifty-three nurses working on the medical and surgical wards at the hospital were invited to participate in the study. However, only 51 were included in the study, as two were not available during the data collection period. A total population sampling strategy was used in order to get adequate findings on the two units occupied by adult inpatients prone to T2DM.

\section{Measures}

A questionnaire was used from a study by Ledbetter entitled "Diabetes understanding among staff nurses: examining the actual versus perceived knowledge in the acute care setting".[19] Permission to use the questionnaire was granted by Ledbetter.[19] The original tool assessed the staff nurses' perceived knowledge of diabetes health education,[19] and the reported Cronbach's value of the original tool was 0.91 .

The tool was adapted with a focus on T2DM, and therefore questions on Type 1 diabetes were removed. The questionnaire had two sections: the sociodemographics, and perception of knowledge and diabetes knowledge questions:

Section A: The Background Data Form had seven questions on the sociodemographic characteristics. Another question related to the amount of diabetes continuing education credits obtained within the last two years was added.

Section B: The Diabetes Basic Knowledge Assessment Tool (DBKT) contained 17 items that assessed the staff nurses' perception of general diabetes knowledge, and specific diabetes health education using the Likert scale format. Scoring ranged from 1 (strongly disagree) to 4 (strongly agree). The tool assessed the diabetes aetiology, pathophysiology of T2DM, basic treatment plans for diabetes, performing blood glucose tests and interpreting blood glucose levels, and management of 'sick day rules' for the diabetic patient.

In addition, the questionnaire contained 27 items that assessed the nurses' knowledge of various content areas with a focus on diabetes health education. The multiple-choice format had three options and a fourth option of "I do not know" was added for those who did not know the test item.

The reported Cronbach's value was 0.85 indicating the tool was reliable. A pilot test was conducted to assess the feasibility of the tool in the CHUB emergency department. The tool was translated into Kinyarwanda, the local language to enhance the understanding and accuracy of participants' responses. The questionnaire scores were converted to percentages and categorised according to Bloom's taxonomy levels of knowledge. The levels were rated as followed: $80-100 \%$ good; $60-$ $79 \%$ moderate; $<60 \%$ poor knowledge. 


\section{Data collection}

After approval from the university and hospital ethics boards, arrangements were made with the Director of Nursing and unit manager to speak to staff in the medical and surgical wards. A meeting was called, and the investigator gave the nurses information about the study and opportunity to participate, knowing that the questionnaire would be coded and did not contain any personal identifying information. Participants signed a consent form and completed the questionnaire in approximately 45-60 minutes. The questionnaire was returned to the investigator on the day it was completed.

\section{Data analysis}

The study used descriptive statistics to analyse the data. Sociodemographic characteristics of participants were measured using frequencies. Knowledge of diabetes health education was measured using frequencies and means. The relationship between the nurses' education level and knowledge of T2DM was calculated using Fisher's exact test, due to the small sample size. A p-value of $<0.05$ was considered significant. Analyses were conducted using SPSS version 23.

\section{Ethical considerations}

Permission was obtained from the University of Rwanda, College of Medicine and Health Sciences (CMHS), Institutional Review Board (IRB). The CHUB ethical research committee approved data collection at the hospital. Consent was obtained from all participants, and no personal identification was linked to the data.

\section{Results}

Fifty-one nurses aged 23 to 52 years, and predominantly female $(58.8 \%)$ participated in the study (table1). The majority was at A1 nurse education level (92.2\%), had $1-5$ years $(68.6 \%)$ nursing experience, and worked on the medical unit $(58.8 \%)$.
Table 1. Socio-demographic characteristics

\begin{tabular}{ll}
\hline Characteristics & $\mathbf{n ~ ( \% )}$ \\
\hline Gender & \\
Female & \\
Male & $30(58.8)$ \\
Age (years) & $21(41.2)$ \\
20-25 & \\
26-30 & $9(17.6)$ \\
$31-35$ & $22(43.1)$ \\
$41-45$ & $14(27.5)$ \\
$>45$ & $3(5.9)$ \\
Nurse education level & $3(5.9)$ \\
A2 & \\
A1 & $2(3.9)$ \\
A0 & $47(92.2)$ \\
Years in Nursing & $2(3.9)$ \\
1-5 & \\
6-10 & $35(68.6)$ \\
$>10$ & $10(19.6)$ \\
Service unit & $6(11.8)$ \\
Medical & \\
Surgical & $30(58.8)$ \\
\end{tabular}

The participants' knowledge of health education for T2DM patients is presented in table 2. Over half $(62.7 \%)$ correctly answered diet questions on nutritional teaching before discharge, though most $(92.2 \%)$ did not know how to calculate a diabetic diet. Nearly half were correct with checking ketonuria $(43 \%)$, effect of stress $(45.1 \%)$, and an action plan if the insulin needle became contaminated (43.1\%).

Table 2. Participants' knowledge of diabetes health education content

\begin{tabular}{llll}
\hline T2DM education & \multicolumn{3}{c}{ Participants' Knowledge } \\
& Correct n (\%) & Incorrect n (\%) & Mean (\%) \\
\hline Monitoring BG & & & 17.6 \\
Accuracy of BG strip preparation & $4(7.8)$ & $47(92.2)$ & \\
Relationship of glycosuria and BG level & $5(9.8)$ & $46(90.2)$ & \\
Checking for ketonuria & $22(43.0)$ & $29(56.9)$ & 31.7 \\
High BG for 2 consecutive days & $5(9.8)$ & $46(90.2)$ & \\
Nutritious meals & $32(62.7)$ & $19(37.3)$ & 31.2 \\
Nutritional teaching before discharge & $4(7.8)$ & $47(92.2)$ & \\
Calculation of diabetic diet & $11(21.6)$ & $40(78.4)$ & 32.4 \\
Developing meal plan & & & \\
Move & $19(37.3)$ & $32(62.7)$ & \\
Effect of exercise on high BG & $23(45.1)$ & $28(54.9)$ & \\
Effect of stress on diabetes & & & \\
Medications & $28(54.9)$ & $23(45.1)$ & $29(56.9)$ \\
Storage of Opened insulin vial & $22(43.1)$ & $2(82.4)$ \\
Action plan if insulin needle contaminated & $9(17.6)$ & $48(94.1)$ & \\
Effect of illness on insulin requirements & $3(5.9)$ & \\
Oral hypoglycemic side effects & &
\end{tabular}

BG Blood glucose 
The majority answered incorrectly questions about selfmonitoring of BG; which included accuracy of strip preparation $(92.2 \%)$, the relationship of glycosuria and BG level $(90.2 \%)$, and meaning of two consecutive days of high BG $(90.2 \%)$ (table 2$)$. The majority also answered medication questions incorrectly, such as the effect of illness on insulin requirements $(82.4 \%)$, and side effects of oral hypoglycemic medications $(94.1 \%)$, though over half $(54.9 \%)$ answered the storage of the insulin vial correctly.

The majority self-reported that they had good knowledge of the action of insulin (83.3\%), sites for the administration of insulin $(88.2 \%)$ and action of oral hypoglycemic agents in T2DM (84.4\%). The majority also self-reported good knowledge of a diet for a T2DM patient $(76 \%)$, and further, the effect of exercise on diabetes control (64.7\%); however, less than half $(39.3 \%)$ of participants self-reported good knowledge of monitoring blood glucose.

The relationship between the level of nurses' education and level of knowledge is presented in table 3. Crosstabulation of the data indicated there was no relationship between the nurses' education and knowledge level of patient education for a T2DM patient $(\mathrm{p}=0.102)$.

Table 3. Relationship between education and knowledge of T2DM

\begin{tabular}{lllll}
\hline $\begin{array}{l}\text { Knowledge } \\
\text { Score (\%) }\end{array}$ & \multicolumn{4}{c}{ Nursing Education Level } \\
& A0 & A1 & A2 & p-value* \\
\hline $33-36$ & 1 & 17 & 2 & 0.102 \\
$37-43$ & 0 & 18 & 0 & \\
$44-48$ & 1 & 12 & 0 & \\
& & & & \\
\hline
\end{tabular}

*Fisher's exact test

The participants' knowledge of the foot care as part of health education for diabetic patients is displayed in figure 1. It was observed that about a half $(50.98 \%)$ knew the diabetic foot-care as a component of health education.

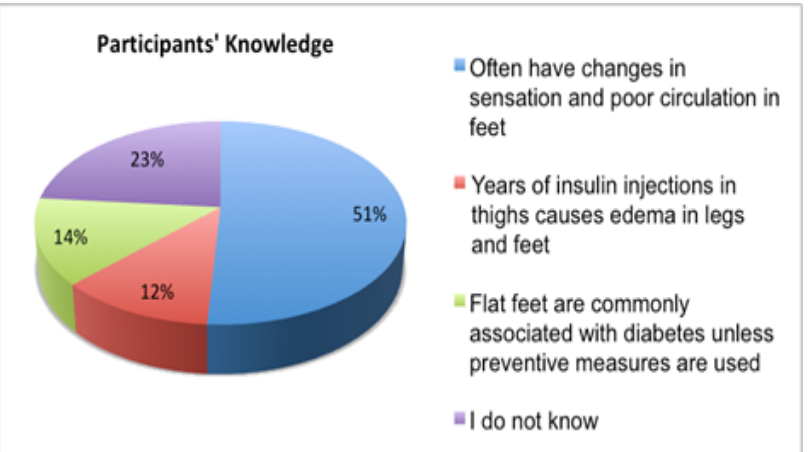

Figure 1. Nurses' knowledge of the foot care as part of health education for diabetic patients

\section{Discussion}

Overall, the knowledge of diabetic care in this study population of 51 nurses at one hospital was very poor, as no nurses cored above $45 \%$. The majority of participants were female $(58.8 \%)$, aged $26-30$ years, A1 nurse education level $(92.2 \%)$, with $1-5$ years of nursing experience, and working on the medical unit $(58.8 \%)$.

The active lifestyle, hilly terrain, and abundance of fresh fruit and vegetables in Rwanda provide a good environment for preventive measures to avoid diabetes. Over $90 \%$ of the population live in the rural areas and are physically active, and would therefore likely be at lower risk for diabetes than their western counterparts.

\section{Monitoring blood glucose}

The majority of participants had a poor knowledge of the factors affecting the accuracy of blood glucose strip, and nearly all $(90.2 \%)$ were not able to recognise the relationship between the value of the urine glucose result and the blood glucose value. This finding indicates that self-blood glucose monitoring among people with diabetes cannot be well taught or remembered, and similar to a study in Nigeria where three quarters $(75.1 \%)$ had poor knowledge of glucose monitoring as part of self-management.[20]

Many HCP are not aware of the different types of diabetes and blood glucose values. Traditionally, there have been two main types of diabetes, Type 1 insulin dependent and T2DM, with gestational diabetes in the second category. In SSA, there are three known subtypes.

The first subtype is similar to insulin resistance of the T2DM seen in Western countries and in urban settings, is related to a BMI higher than normal $(>25 \mathrm{~kg} / \mathrm{m} 2)$, high calorie diet, sedentary lifestyle, and greater waist circumference, with women $(>80 \mathrm{~cm})$ and men $(>90$ $\mathrm{cm}$ ), and a metabolic syndrome involving HTN and high cholesterol.[5,7,20]

The second subtype of T2DM is related to malnutrition of people in rural environments with normal or underweight $(\mathrm{BMI} \leq 25)$ status, severe hyperglycemia and typically without ketonuria. The extended malnutrition likely results in pancreatic calcification. $[5,6]$

The third subtype of T2DM has a similar presentation to type 1 diabetes, with severe hyperglycemia, weight loss, ketonuria, polyuria, and polydipsia. $[5,6]$

\section{Move}

Participants were unable to link the physical activity to diabetes control as over half $(54.9 \%)$ answered the question incorrectly. The majority (62.7\%) appeared less knowledgeable about the effect of physical exercise on high blood glucose, similar to a quarter $(24.9 \%)$ of participants in a study conducted in Nigeria,[20] and others in the UK,[21] and Brazil.[22] The strategy 
of using physical exercise is to lose weight or maintain weight at a normal BMI $(<25 \mathrm{~kg} / \mathrm{m} 2)$, and lower the risk of T2DM; however, this might apply to urban populations consuming high-calorie diets and needing to adjust their lifestyle. Rwandans who live in the rural area are physically active on a daily basis and therefore unlikely to lead a sedentary lifestyle, or have a high-calorie diet.

\section{Nutritious meals}

The findings revealed that the majority $(69.3 \%)$ did not perform well on questions related to the impact of nutrition in T2DM self-management. Most Rwandans live in rural areas and primarily consume fresh local produce, though the low diabetes prevalence rate of $3.2 \%$ will likely increase in the future with more urbanization and the attendant high-fat and high-sugar diets. A nutritional and well-balanced diet constitutes one of the survival skills for a diabetic patient; therefore, it is almost impossible to expect positive outcomes, if patients are not aware of meals to consume. Less than $10 \%(7.8 \%)$ of participants knew how to calculate dietary needs for diabetic patients. These findings are similar to another study that revealed that $44 \%$ demonstrated poor knowledge of basic diabetic meal planning, and over $70 \%$ had never provided dietary advice to diabetic patients.[9]These results indicated a need for hospitals to develop diabetic teaching protocols and mandatory annual continuous professional development (CPD).

\section{Medication}

The majority $(78.95 \%)$ of participants had poor knowledge of medications used for diabetic care. Nearly half $(45.1 \%)$ did not know where to store an insulin vial, similar to another study that found over half $(60.15 \%)$ had not answered a general insulin question correctly, and less than half $(39.79 \%$ ) were knowledgeable about insulin therapy for children.[24] Additionally, nearly all $(94.1 \%)$ demonstrated poor knowledge regarding oral hypoglycemic agents, a group of drugs that are commonly used among patients with T2DM.

Proper storage of insulin is essential as the medication is sensitive to sunlight, artificial light, and high temperatures, whether they be hot or freezing cold.[25] The guidelines stipulate the following:

- An UNOPENED insulin vial is best stored inside the refrigerator at $\left(2^{\circ}\right.$ to $8^{\circ}$ Celsius). It is good in the refrigerator until the expiration date on the box.[25]

- An OPENED insulin vial means the insulin cap has been removed and the rubber stopper has been punctured with a needle. OPEN vials can be kept at room temperature $\left[15-25^{\circ} \mathrm{Celsius}\right]$ or in the refrigerator for 28 days. [25]

\section{More}

Most participants were knowledgeable of the importance of foot care for diabetic patients and its prevention, as half $(50.98 \%)$ were knowledgeable of poor sensation and circulation related to diabetic foot. According to Bloom's classification of moderate knowledge, they still did not attain the level of moderate knowledge, similar to a study in Nigeria that indicated poor knowledge.[20] In contrast, a study conducted in Saudi Arabia revealed that nurses had good knowledge of diabetic foot care, but they had a poor practice of preventing complications.[23]

The findings of this study showed that there was no relationship between the level of education and level of knowledge among nurses delivering health education toT2DM patients $(p=0.102)$. A UK study aimed at the "Assessment of diabetes knowledge in trained and untrained ward nurses before and after intensive specialist support" revealed that nurses with inadequate knowledge of diabetes management improved with education and support,[25] in particular, the timing and administration of insulin, use of metformin in renal impairment, and when to escalate blood ketone results.[25]

The findings of an integrative review of the international nursing workforce reported knowledge deficits of diabetes care.[16] The results of the review and our study findings, considering the likely increased prevalence of T2DM worldwide, indicates a need for more importance placed on diabetes education in nursing school and regular, affordable CPD and training at work. The limited number to diabetic experts, a shortage of nurses in certain health facilities, and heavy patient workloads, with subsequent low staff morale, creates many barriers to improve the situation of diabetic care. Nurses should be encouraged to become experts in diabetes care and conduct original research or translational research as part of a multidisciplinary team. There is a great need within Africa to conduct regional research due to the limited research currently available to help guide practice.[6]

\section{Limitations}

This study had a small sample size and limited to only one hospital in Rwanda; therefore it cannot be generalised to other facilities or nationwide.

\section{Conclusion}

The nurses that participated in this study exhibited poor knowledge concerning T2DM health education content, similar to an international integrative review. With the increasing prevalence of T2DM, healthcare professionals will need to improve their health education information they give to patients.

\section{Acknowledgements}

The authors are thankful to Dr Pamela Meharry for support with this manuscript.

This article is published open access under the Creative Commons Attribution-NonCommercial NoDerivatives (CC BY-NC-ND 4.0). People can copy and redistribute the article only for noncommercial purposes and as long as they give appropriate credit to the authors. They cannot distribute any modified material obtained by remixing, transforming or building upon this article. See https:// creativecommons.org/licenses/by-nc-nd/4.0/ 


\section{References}

1. International Diabetes Federation. Diabetes Atlas [Internet]. Eight Edit. 2017. Available from: https:// diabetesatlas.org/resources/2017-atlas.html

2. World Health Organization. Diabetes fact sheets. 2018; Available from: https://www.who.int/ news-room/fact-sheets/detail/diabetes

3. Rwanda Diabetes Association. Health magazine. 2015;(05):1-16. Available from: http:/ / rwandadiabetes.com/wp-content/ uploads/2016/01/Eureka_5.pdf

4. World Health Organization. Noncommunicable diseases fact sheets. 2018; Available from: https:/ / www.who.int/news-room/fact-sheets/detail/ noncommunicable-diseases

5. Atun R, Davies JI, Gale EAM, Bärnighausen T, Beran D, Kengne AP, et al. Diabetes in sub-Saharan Africa: From clinical care to health policy The Lancet Diabetes \& Endocrinology Commission Diabetes in sub-Saharan Africa : from clinical care to health policy. 2017;8587(December).

6. Pastakia, SD, Pekny, CR, Manyara, MS, and Fischer L. Diabetes in sub-Saharan Africa - from policy to practice to progress: targeting the existing gaps for future care for diabetes. 2017;247-63.

7. Hall V, Thomsen RW, Henriksen O, Lohse N, Sicree R, Shaw J, et al. Diabetes in Sub Saharan Africa 1999-2011: Epidemiology and public health implications. a systematic review. BMC Public Health [Internet]. 2011;11(1):564. Available from: http://bmcpublichealth.biomedcentral.com/ articles/10.1186/1471-2458-11-564

8. Ulrich PA, Abner N. Diabetes under control: Meter, Meds, Meals, Move, and More. Am J Nurs, 2010;110(7):62-5. doi: 10.1097/01. NAJ.0000383938.76085.00.

9. Mogre V, Ansah G a., Marfo DN, Garti Ha. Assessing nurses' knowledge levels in the nutritional management of diabetes. Int J Africa Nurs Sci [Internet]. 2015;3:403. Available from: http://linkinghub.elsevier.com/ retrieve/pii/S2214139115000177

10. Breen C, Ryan M, Gibney MJ, Shea DO. Diabetesrelated nutrition knowledge and dietary intake among adults with type 2 diabetes Diabetesrelated nutrition knowledge and dietary intake among adults with type 2 diabetes. 2015; (July).

11. Zeb A, Khan M, Wahab F, Khan MT, Nawaz A. Knowledge Attitude and Practice of Diet and Exercise among Diabetic Patients for Normal Plasma Glucose. 2017;7(1):131-5.

12. Glabska D, Wlodarek D. Analysis of the declared nutritional behaviours in a group of diabetology nurses educating patients about diabetes diet therapy. Rocz Panstw Zakl Hig. 2015;66(4):345-51.

13. Ufashingabire CM, Nsereko E, Njunwa KJ, Brysiewicz P. Knowledge and Attitudes of Nurses
Regarding Pain in the Intensive Care Unit Pa tients in Rwanda. 2016;3(1):21-6.

14. Manwere A, Chipfuwa T, Mukwamba, Maceline Mutsa and Chironda G. Knowledge and Attitudes of Registered Nurses towards Pain Management of Adult Medical Patients: A Case of Bindura Hospital. 2015;1-6.

15. Bilal M, Haseeb A, Rehman A, Arshad MH, Godil S, Qamar MA, et al. Knowledge, Attitudes, and Practices Among Nurses in Pakistan Towards Diabetic Foot. 2018;10(7).

16. Alotaibi A, Al-ganmi A, Gholizadeh L, Perry L. Diabetes Knowledge of Nurses in Different Countries: An Integrative Review. Nurse Educ Today [Internet]. 2016;(December 2017). Available from: http://dx.doi.org/10.1016/j. nedt.2016.01.017

17. East S, Okpala PU, Uwak R, Nwaneri AC, Onyiapat J, Emesowum A, et al. Nurses' knowledge and attitude to the care of HIV / AIDS patients in. 2017;4(2):547-53.

18. World Health Organization. Diabetes in SouthEast Asia: burden, gaps, challenges and ways forward. 2016;5(2):77-173.

19. Ledbetter RB, Carlton JM. Diabetes Understanding Among Staff Nurses: Examining The Actual Versus Perceived Knowledge In The Acute Care Setting. 2011;

20. Oyetunde MO, Famakinwa T'T. Nurses' knowledge of contents of diabetes patient education in Ondo - state, Nigeria. J Nurs Educ Pract [Internet]. 2014;4(4). Available from: http://www.sciedu.ca/ journal/index.php/jnep/article/view/2779

21. Matthews A, Jones N, Thomas A, Van Den Berg P, Foster C. An education programme influencing health professionals to recommend exercise to their type 2 diabetes patients - Understanding the processes: A case study from Oxfordshire, UK. BMC Health Serv Res [Internet]. 2017;17(1):1-15. Available from: http://dx.doi.org/10.1186/s12913-017-2040-7

22. Burdick L, Mielke GI, Parra DC, Gomes G, Florindo A, Bracco M, et al. Physicians', nurses' and community health workers' knowledge about physical activity in Brazil: A cross-sectional study. Prev Med Reports [Internet]. 2015;2:467-72. Available from: http://dx.doi.org/10.1016/j. pmedr.2015.06.001

23. Abdullah WH, Senany $\mathrm{S} \mathrm{Al}, \mathrm{Al}$-otheimin $\mathrm{HK}$. Capacity Building for Nurses' Knowledge and Practice Regarding Prevention of Diabetic Foot Complications. 2017;7(1):1-15.

24. Chrysoula D, Victoria A, Anna P, Eirini M, Nikoletta MM, Sofia Z. Assessment of insulin treatment - related knowledge among nurses in Pediatric Hospitals. 2016;6(4):20-5.

25. Consumer Med Safety. Storage of Insulin, 2018. Available from: https://www.consumermedsafety.org/ 